\title{
LETTER
}

\section{Hypercapnia and hypokalemia in near-death experiences}

\author{
Bruce Greyson* \\ See related research by Klemec-Ketis et al., http://ccforum.com/content/14/2/R56
}

Klemenc-Ketis and colleagues' novel report of hypercapnia and hypokalemia associated with near-death experiences (NDEs) [1] was somewhat surprising, as Sabom [2] had previously reported lower than normal carbon dioxide levels measured at the time of a patient's NDE, and Parnia and colleagues, in a prospective study of 63 cardiac arrest survivors, had found no significant association of either potassium or carbon dioxide with NDEs [3].

Klemenc-Ketis and colleagues' conclusion that hypercapnia plays a role in provoking NDEs is one possible interpretation of the correlation they found. It is also plausible that hypercapnia is simply an indicator of another factor that may be linked causally to NDE reports. For example, the authors noted that hypercapnia indicates better cardiac output and perfusion pressure, which would reduce the amnesia that is usually seen in cardiac arrest, so that patients would be more likely to remember what happened during the arrest. The association between NDEs and hypercapnia may thus indicate simply that patients who are able to recall more of their cardiac arrest also report more NDEs. Gliksman and Kellehear reviewed studies showing that levels of carbon dioxide in the blood are not necessarily accurate estimates of levels in the brain [4], which further complicates the interpretation of the current findings.

The small sample size of this study, the contradictory evidence from other studies, and the unclear association between levels of carbon dioxide in the blood and in the brain suggest caution in interpreting the findings and suggest the need for further research.

\section{Authors' response}

Zalika Klemenc-Ketis, Janko Kersnik and Stefek Grmec

Bruce Greyson proposes an alternative explanation for the results of our study [1], which can also be plausible. Namely, higher levels of the partial pressure of end-tidal carbon dioxide (petCO $\mathrm{C}_{2}$ ) are also indicators of better cardiac output, as discussed in our article [1] and confirmed in our previous studies [5]. Higher incidence of reported NDEs in patients with hypercapnia might therefore indicate simply better memory of the actual NDE event, but this should be confirmed with further studies.

A study about the effects of meditation on respiration and temporal lobes indicated that higher partial pressure of carbon dioxide $\left(\mathrm{pCO}_{2}\right)$, which is a result of special breathing techniques during meditation, might have been important in provoking cognitive and emotional changes

*Correspondence: cbg4d@virginia.edu

Department of Psychiatry \& Neurobehavioral Sciences, University of Virginia Health System, $21010^{\text {th }}$ Street NE, Charlottesville, VA 22902-5328, USA
[6]. Also, higher levels of $\mathrm{pCO}_{2}$ presumably have an excitatory effect on the limbic system, which might result in mystical (NDE-like) experiences [6]. The possible connection between the limbic system and NDE-like experiences has already been reported [7].

Higher petCO $\mathrm{C}_{2}$ and $\mathrm{pCO}_{2}$, besides better cardiac output, might therefore indicate also a possible connection between carbon dioxide and the incidence of NDEs. Since patients with asphyxia cardiac arrest were found to have higher pet $\mathrm{CO}_{2}$ than patients with primary cardiac arrest [8], the patients with asphyxia cardiac arrest might also have higher incidence of NDEs - which, if confirmed, might help to clarify the role of carbon dioxide in NDEs. This theory should be further investigated in larger and multicentre studies, but in the light of patient-oriented care it is important to take into account the existence of NDEs in cardiac arrest patients and to develop protocols of care for such patients.

\section{Abbreviations}

$\mathrm{NDE}$, near-death experience; $\mathrm{pCO}_{2}$, partial pressure of carbon dioxide; pet $\mathrm{CO}_{2}$, partial pressure of end-tidal carbon dioxide. 


\section{Competing interests}

The author declares that he has no competing interests.

Published: 27 May 2010

\section{References}

1. Klemenc-Ketis Z, Kersnik J, Grmec S: The effect of carbon dioxide on neardeath experiences in out-of-hospital cardiac arrest survivors: a prospective observational study. Crit Care 2010, 14:R56.

2. Sabom MB: Recollections of Death: A Medical Investigation. New York: Harper and Row; 1982.

3. Parnia S, Waller DG, Yeates R, Fenwick P: A qualitative and quantitative study of the incidence, features and aetiology of near death experiences in cardiac arrest survivors. Resuscitation 2001, 48:149-156.

4. Gliksman MD, Kellehear A: Near-death experiences and the measurement of blood gases. J Near-Death Studies 1990, 9:41-43.
5. Kolar M, Krizmaric M, Klemen P, Grmec S: Partial pressure of end-tidal carbon dioxide successful predicts cardiopulmonary resuscitation in the field: a prospective observational study. Crit Care 2008, 12:R115.

6. Petersson Bouin Y: Effects of Meditation on Respiration and the Temporal Lobes: An Exploratory and Meta-analytic Study. Lund: Lund University Press; 2000.

7. Britton WB, Bootzin RR: Near-death experiences and the temporal lobe. Psychol Sci 2004, 15:254-258.

8. Grmec S, Lah K, Tusek-Bunc K: Difference in end-tidal $\mathrm{CO}_{2}$ between asphyxia cardiac arrest and ventricular fibrillation/pulseless ventricular tachycardia cardiac arrest in the prehospital setting. Crit Care 2003, 7:R139-R144.

doi:10.1186/cc9016

Cite this article as: Greyson B: Hypercapnia and hypokalemia in near-death experiences. Critical Care 2010, 14:420. 